\title{
LA ROYAUTÉ FRANÇAISE ENTRE FÉODALTTÉ ET SACERDOCE ROI SEIGNEUR OU ROI MAGISTRAT?
}

Le roi est-il le premier magistrat ou est-il le premier seigneur du royaume? Sur ce vieux canevas, René-Louis de Voyer d'Argenson, le secrétaire d'État disgrâcié, brodait des variations plus originales qu'il y paraît :

"La Monarchie proprement dite s'entend d'un Gouvernement où le Monarque rapporte tout à lui, ne considérant en cela que son droit de propriété sur les États qu'il gouverne [...] La Royauté est le Gouvernement d'un État par un homme seul qui considère moins son droit de propriété que le bien de l'État qu'il gouverne et dont il ne se regarde que comme le premier Magistrat " (Considérations sur le gouvernement ancien et présent de la France publiées en $\left.1765^{*}\right)$.

Le roi magistrat pouvait se définir par ses devoirs, le roi seigneur par ses droits. Ces deux figures de la monocratie roulaient depuis le Moyen Âge sur deux pôles : l'un d'origine cléricale, c'était le concept d'office comme dignitas; l'autre d'origine féodale, c'était la rationalisation du dominium seigneurial. Réinterprétant le droit romain, la tradition bartoliste, avec beaucoup de savoir, avait légué la double conception d'un imperium et d'un dominium divisés sur le même modèle. La science politique classique (Charles Loyseau en offre un bon exemple) prêta aux Romains une conception unitaire de l'imperium et du dominium et, théorisant la pratique de la monarchie moderne, réunifia l'imperium (quand elle ne le dépassa pas, comme Bodin), tout en reconnaissant l'éclatement du dominium ${ }^{1}$. Le mouvement s'accompagna d'une critique sévère -

\footnotetext{
* Pour diminuer le nombre des notes, on a signalé dans le cours du texte les références des passages tirés des jurisconsultes d'Ancien Régime: la consultation des éditions modernes ou du catalogue des livres imprimés de la Bibliothèque nationale suffira à les repérer précisément.

1. Édouard Maynial, "Notes sur la formation de la théorie du domaine divisé (domaine direct et domaine utile) du $x_{11}{ }^{c}$ au xIv siècle dans les Romanistes. Étude de dogmatique juri-
}

Revue de synthèse : IV S. N ${ }^{\text {os }} 3-4$, juil.-déc. 1991. 
mais inadéquate? - de la féodalité. L'enjeu se mesure à l'âpreté des polémiques qui opposèrent les juristes humanistes autour de la définition de l'imperium romain (Le Duaren, Govean, Cujas) et à la violence du refus que suscita leur démarche chez les jurisconsultes qui, comme Hotman, Bodin ou Loyseau, étaient désireux d'élaborer une théorie neuve de la monarchie française. Roi magistrat ou roi seigneur? À la Renaissance, le débat prit l'ampleur de «l'État d'offices " en construction, car la question des droits du roi sur la royauté et celle des droits de l'officier sur son office renvoyèrent bientôt en miroir l'une à l'autre. Charondas Le Caron, dans ses Pandectes, ne voyait-il pas dans le roi le "patron et modèle" "sur lequel tous magistrats dressent les desseins et projets de leur charges et offices "?

Toutefois la pensée politique de la Modernité, suivant en cela la pratique, ne partit pas d'un postulat d'exclusion entre le roi magistrat ou le roi seigneur; elle pensa au contraire les deux propositions en termes de relation. C'est ce que se sont souvent refusés à faire les historiens français du droit, privilégiant unilatéralement la théorie du roi officier :

"Il est donc de principe, écrivait Gabriel Lepointe, que le Roi est distinct de la Couronne qui le transcende et ceci érige la royauté en institution de droit public, écartant toute idée de droit privé, toute prétention patrimoniale. On rejoint par là la notion de monarchie pure : car le roi, n'étant nullement propriétaire du royaume, n'est pas davantage maitre des biens de ses sujets " ${ }^{2}$.

Cet amalgame déductif, hérité de l'enseignement éminent de François Olivier-Martin et de Joseph Declareuil, reflète l'apologétique monarchique développée avec tant de talent par les jurisconsultes de l'Ancien Régime, il néglige la construction de leur raisonnement. Nous pressen-

dique ", Mélanges pour les soixante-quinze ans du professeur Hermann Fitting, Montpellier, 1908, p. 409-461. Vincenzo PIANo MoRTARI, Il Potere sovrano nella doctrina giuridica del secolo $x V$, Naples, Jovene, 1973. Dans la bibliographie immense qui traite de la naissance d'une science politique moderne, on se référera simplement à Donald R. KeLLEY, Foundations of Modern Historical Scholarship : Language, Law and History in the French Renaissance, New York, Columbia University Press, 1970; John G. A. Pocock, The Ancient Constitution and the Feudal Law, Cambridge, Cambridge University Press, 1957; Julian H. Franklin, Jean Bodin and the Rise of Absolutist Theory, Cambridge, Cambridge University Press, 1973; Howell A. LLoYd, "The Political Thought of Charles Loyseau (15641627) ", European Studies Review, vol. XI, 1981, p. 53-82.

2. Gabriel Lepolnte, "Le régime monocratique dans les trois derniers siècles de l'ancienne France ", in La Monocratie, Bruxelles, Ed. de la Libr. encyclopédique, "Recueils de la Société Jean Bodin pour l'histoire comparative des institutions ", XXI, 1969, p. 680. On assiste aujourd'hui à la fin du « consensus historiographique, élaboré depuis plus d'une génération » par l'école française d'histoire du droit dont François Olivier-Martin apparaît comme le maître le plus représentatif ; cf. Gérard GIORDANENGO, " Le pouvoir législatif du roi de France $\left(\mathrm{Xr}^{\mathrm{e}}\right.$-xII $\mathrm{e}^{\mathrm{e}}$ siècle) : travaux récents et hypothèses de recherche ", Bibliothèque de l'École des Chartes, t. 147, 1989, p. 284-285. 
tons aujourd'hui que la distinction entre le roi et la couronne s'est faite évanescente au fur et à mesure que la "monarchie pure " de la Renaissance évoluait vers la monarchie absolue. Il faut considérer comme problématiques des propositions telles que la royauté n'a aucun caractère patrimonial, ou bien le roi n'est pas propriétaire de son royaume et, a fortiori, il n'est pas maître des biens de ses sujets. Problématiques, non pas erronées...

L'articulation entre la puissance et la propriété du roi et de ses officiers ne soulève pas une simple question doctrinale, elle conditionne aussi l'exercice du pouvoir concernant des droits si fondamentaux que l'impôt et le consentement, que la propriété et le respect des contrats. En ce sens, l'imperium et le dominium romanistes restèrent l'horizon indépassé du droit public monarchique.

Les anciens juristes aimaient à expliquer que la « loi et coutume du royaume " faisait échapper la dévolution de la couronne à l'hérédité stricte. Cette doctrine restait dominante même à l'époque où triomphait la mystique bourbonienne du sang royal : aucune analogie n'était dès lors légitime entre la propriété privée et le domaine public. Dans ses Traités touchant les droits du Roi très-chrétien..., vers 1630 (?), publiés en 1655 et 1670, l'érudit Pierre Dupuy avertissait :

«Il faut considérer qu'il n'en est pas des Rois comme des particuliers. Les particuliers disposent de leurs biens [...], les font passer à leurs héritiers aux charges qu'il leur plait. Les Rois, au contraire, n'ont pas la libre disposition d'aucune partie du Domaine de la Couronne, ils sont usufruitiers, sont administrateurs, sont comme les tuteurs de leurs Royaumes. "

Jean Savaron rappelait aussi en 1620 que «le roi n'est seulement qu'administrateur et comme usufruitier de son royaume " et que "le Tout-Puissant, le Roi des Rois, Souverain des Souverains " a établi le roi " son vicaire au temporel " du royaume. Un discours parallèle était tenu par les parlementaires depuis la fin du $x v^{e}$ siècle au moins.

L'origine de ces théories est à chercher dans les Tractatus de Jean de Terrevermeille. Sans doute est-ce Charles Du Moulin qui fit le plus pour populariser l'adage « rex non censetur dominus seu proprietarius regni sui, sed administrator ». Cet aphorisme procédait chez lui d'une vision cohérente de l'autorité, qui s'appliquait au pouvoir pontifical ( "le pape n'est seigneur de l'Église, ains seulement commis et serviteur ", Abus des petites dates, 1552-1564), comme à l'autorité des magistrats ( «les juges ordinaires ne sont pas seigneurs de leur juridiction, mais simples administrateurs", citation de Balde que Du Moulin commentait en ces termes : " celui qui détient un office n'a aucun droit sur la juridiction, aucune possession, mais le nu exercice et l'administration au nom du sei- 
gneur (" nomine domini")" (Commentaire sur la coutume de Paris, 1539). Tous les magistrats, depuis le roi jusqu'au juge inférieur, exerçaient leur pouvoir à un titre analogue, qui est comparé à un «usufruit " $"$

Cette analogie empruntée au droit privé semble supposer un propriétaire de la prérogative publique : pour les offices, il est loisible d'imaginer avec le maître de Du Moulin, André Alciat (1492-1550), que le prince s'est réservé la seigneurie de l'office en en concédant l'exercice. Mais qu'en est-il de l'office du prince? Trois réponses étaient concevables : Dieu, le peuple, l'État. Pour Bodin, «il est sans difficulté que tous les états, magistrats et offices appartiennent à la République en propriété » (République, III, 5), solution théorique également adoptée par Charles Loyseau. Mais, derrière l'État, se profilait la communauté du royaume : nul ne contestait sérieusement la démonstration de François Hotman quant aux droits originels des Francs-Gaulois, au moins jusqu'aux thèses " romanistes " popularisées par l'Histoire critique de l'établissement de la monarchie française dans les Gaules (1719) de l'abbé Jean-Baptiste Dubos, qui soutenait que les rois mérovingiens avaient directement succédé aux pouvoirs de l'empereur romain en Gaule.

L'image de l'« usufruit " recelait quelques dangers pour une pratique absolue de la monarchie. En fait, Du Moulin et probablement Alciat restaient en partie prisonniers des conceptions des glossateurs et des postglossateurs qu'ils critiquaient. Bartole attribuait un imperium particulier à chaque magistrat dans sa sphère propre ; à ses yeux, les diverses universitates étaient souveraines pour leurs affaires particulières suivant le principe de la civitas sibi princeps. Presque deux siècles auparavant, Azon, dans sa célèbre controverse avec Lothaire - qui servait encore de point de départ à la réflexion de Bodin et de Loyseau ! - , avait soutenu que le roi et ses officiers partageaient le merum imperium. Le concept platonicien d'émanation permettait bien à $\mathrm{Du}$ Moulin de penser la délégation du pouvoir que le roi confiait en dépôt au magistrat. Mais l'ancienne représentation de l'arbor imperiorum demeurait vivante jusque chez Bodin et ses contemporains (Traité des offices de Charles de Figon en 1579). Il existait donc une cohésion fonctionnelle de la magistrature tant que le monarque demeurait le justicier suprême et non le Pygmalion de la loi. Bodin s'étonnait de trouver un élu du xive siècle qui se proclamait "Élu par la grâce de Dieu », fait que conteste Charles Loyseau.

L'idée de faire du roi l'usufruitier de la couronne participait d'une vision traditionnelle qui aboutissait à limiter la puissance souveraine en instaurant une transcendance abstraite au-dessus du souverain concret. La notion d'office débouchait sur celle de sacerdoce, conférant au roi, 
mais aussi aux magistrats, une quasi-sacralité ${ }^{3}$. Au temps des guerres de Religion, c'était faire le jeu des Monarchomaques, qui, tel Théodore de Bèze (1519-1605), dans $D u$ droit des magistrats (1574), parlaient de magistrats « établis protecteurs des droits de la souveraineté pour retenir le souverain en son devoir ", " encore qu'ils n'aient la puissance souveraine et ordinaire à manier ", car " leur devoir est de garder les bonnes lois, desquelles ils ont juré l'observation à l'encontre de tous, selon la portion de l'état public qui leur est commise ". Il était logique que la polémique protestante se livrât à une déconstruction rationnelle de la notion de dignitas qui avait autorisé le transfert sur la royauté des qualités " épiscopales " que suggérait la notion d'usufruit appliquée aux communautés ecclésiastiques puis laïques.

Cependant, à partir du xvII siècle, une autre solution semble avoir rencontré les faveurs des juristes : image bizarre, empruntée comme celle de l'usufruit au droit privé, c'est le fidéicommis. Charles Loyseau pensait que la loi salique "induit une manière de substitution graduelle en la famille des princes du sang, ni plus ni moins que nos lois disent des fidéicommis laissés aux familles " (Offices, II, 2). Un siècle plus tard, le duc de Saint-Simon comparait également la couronne à « un fidéicommis qui ne lui appartient pas en propre [au roi] et dont il ne peut disposer", l'ayant reçue « de ses pères à titre de substitution et non pas de libre héritage ". Dans l'Essai d'une institution au droit public, publié en 1759, le chancelier d'Aguesseau déclarait que, "par une espèce d'inféodation faite en la faveur de la famille dominante, Dieu veut bien transmettre la puissance royale de génération en génération à l'aîné de cette famille ". Cette théorie était encore développée à la veille de la Révolution. Mais, ainsi assimilé à une sorte de majorat, le royaume, quoique soumis à des règles dérogatoires du droit successoral commun, relevait de la catégorie de propriété : une forme archaïque de propriété familiale qu'on pourrait juridiquement rapprocher des fiefs masculins (à cause du service ou de la dignité ?), ou, mutatis mutandis, de ces biens propres qui étaient la propriété morale des lignages. Au demeurant, il faut souligner que l'image du fidéicommis ne s'appliquait pas initialement au royaume, mais au domaine royal : le roi, écrivait René Choppin, " est tenu et obligé de laisser le domaine de France entièrement à son successeur comme par droit de fidéicommis, afin qu'il ne soit blâmé d'être perfide et déloyal » (Trois

3. Cet aspect perdurable de l'idéologie monarchique est souligné par Marc Fumarol, "Sacerdoce et office civil : la monarchie selon Louis XIV ", in Les Monarchies, Emmanuel Le Roy Ladurie, éd., Paris, Presses universitaires de France, 1986, p. 101-114. La question est traitée dans toute son ampleur par Ernst Kaniorowicz, Les Deux corps du roi, 1957, Paris, Gallimard, 1989 et par Marie-France Renoux-Zagame, Origines théologiques $d u$ concept moderne de propriété, Genève, Droz, 1987. 
Livres du domaine, 1572, trad. franç. 1612). L'extension de la métaphore ne participait-elle pas du désir de faire échapper la monarchie à ses fondements féodaux?

Dans l'esprit de ses promoteurs, la théorie du roi officier n'était pas vraiment exclusive d'une conception propriétaire de la royauté. Terrevermeille, qui écrivait pour prouver que Charles VI n'avait pas le droit de déposséder le dauphin, traitait du dominium du royaume. Son raisonnement reposait sur la comparaison du fils dans la domus et du dauphin dans le royaume. Il construisait ainsi le dominium du dauphin sur le modèle de l'heres suus, en en éliminant naturellement la patria potestas qui constituait l'antithèse exacte du but de sa démonstration. Le concept n'est donc pas univoque, ni la relation réversible. Mais il reste clair que l'administratio ou le regimen regni découle du dominium et que le droit du dauphin, dominus en second, coexiste avec celui du roi qui ne saurait l'abolir. Malgré des modalités différentes, un déterminisme héréditaire régit le dominium privé et le dominium royal ${ }^{4}$.

Du Moulin, confronté au délicat problème de la minorité du prince, recourait également à la notion de dominium :

« Nonobstant, écrivait-il, en concédant et transmettant le royaume à un mineur, il semble qu'on lui confère aussi la juridiction [...], ce qui est vrai quant à la propriété, au droit et à la seigneurie (hoc verum est quantum ad proprietatem, jus et dominium) de la juridiction [...], mais non quant à son administration et exercice. "

Il ne faisait aucun doute pour le jurisconsulte gallican que le roi jouissait du "dominium directum et jus regium universaliter in toto regno" (Commentaire sur la coutume de Paris, 1539)'.

La République de Bodin est parcourue par l'analogie entre la propriété du père de famille et la souveraineté du roi : « les chefs de famille ont le gouvernement de ce qui leur est propre ", laissant à la République le gouvernement "de ce qui leur est commun" (République, I, 2). Bodin a même souligné (ibid., 1,8$)$ la relative incompatibilité qui existe entre la souveraineté et la doctrine du roi officier :

" Si la puissance absolue lui est donnée purement et simplement sans qualité de magistrat, ni de commissaire, ni forme de précaire, il est bien certain que

4. Voir Jean Barbey, La Fonction royale. Essence et légitimité d'apress les "Tractatus" de Jean de Terrevermeille, Paris, Nouvelles éd. latines, 1983, en part. p. 338-346 et 361 .

5. Voir Jean-Louis Thireau, Charles Du Moulin, Genève, Droz, 1980. Les controverses autour du problème de la minorité sont exposées par Arlette Jovanna, Le Devoir de révolte. La noblesse française et la gestation de l'État moderne (1559-1661), Paris, Fayard, 1989, p. 334-340. 
cestui-là est et se peut dire monarque souverain : car le peuple s'est dessaisi et dépouillé de sa puissance souveraine pour l'ensaisiner et investir [...] comme celui qui a donné la possession et propriété de ce qui lui appartenait. 》

Naturellement, la métaphore de la propriété n'implique nullement chez Bodin que la royauté ne soit pas un "office " au sens stoïcien et chrétien ${ }^{6}$.

Il revenait à Charles Loyseau de donner une synthèse qui échappait à l'obscurité des analogies implicites. Pour lui, la royauté, telle la pairie, se définissait comme « office seigneurial » : "le roi est parfaitement officier, ayant le parfait exercice de toute-puissance, et est aussi parfaitement seigneur, ayant en perfection la propriété de toute-puissance publique " (Offices, II, 2). Ce serait une grave erreur d'appliquer aux rois de France la définition du " simple prince » qui, non souverain, est seulement officier. Mais la pensée de Loyseau ne s'arrêtait pas à cette étape : « néanmoins il est vrai que les rois ont encore plus de marques et propriétés d'officiers que de seigneurs. " Cette proposition constituait une application d'une règle de la logique aristotélicienne : « l'accessoire suit le principal. » Dans la royauté, l'office était le principal, la seigneurie l'accessoire; aux origines, le fief avait eu une structure identique, mais l'usurpation féodale avait renversé les termes et attaché l'hérédité acquise par le fief à l'office qui y était associé. La royauté aurait-elle conservé la pureté primitive de "l'office seigneurial »? On connaît les célèbres définitions qu'a données Loyseau de l'office («dignité avec fonction publique ") et de la seigneurie (" dignité avec puissance publique en propriété $»)$. Cette dernière définition donnait lieu à la distinction capitale entre "seigneurie privée ", qui est le dominium latin, et la "seigneurie publique ", qui est l'imperium. Ces deux seigneuries ne sauraient se confondre : elles sont « entièrement différentes quant à l'effet ", car " la seigneurie publique [...] n'attribue aucune seigneurie privée ». Mais une autre distinction venait se superposer à la première : l'opposition entre la " seigneurie in abstracto" (" puissance propriétaire ") et la " seigneurie in concreto" (" terre seigneuriale "). En effet, "la seigneurie prise in concreto est comme formée et créée de la seigneurie publique et de la seigneurie privée ». Dans sa complexité, la leçon de Loyseau introduit une clarté (fictive ?) là où régnait la confusion. Le royaume réalisait la fusion légitime de la seigneurie publique et de la seigneurie privée, il était à ce

6. Une telle doctrine a des fondements anti-artificialistes : voir Michel WALZER, La Révolution des Saints, 1965, Paris, Belin, 1987 ou Gordon J. Scochet, Patriarchalism in Political Thought. The Authoritarian Family and Political Speculation and Attitudes..., Oxford, Basil Blackwell, 1975. 
titre une seigneurie in abstracto et in concreto qui absorbait toutes les autres seigneuries in concreto. Le mouvement de la théorie venait ici au secours du mouvement de l'histoire : l'intégration des principautés féodales au domaine royal sanctionnait la fin de cette « absurdité » qu'était l'appropriation privée de la seigneurie publique. Loyseau instituait la royauté en unique féodalité légitime. Car la seigneurie royale ne faisait pas exception à la règle : «comme c'est le propre de toute seigneurie d'être inhérente à quelque fief ou domaine, aussi la souveraineté in abstracto est attachée à l'État » (cf. Seigneuries, I, II et IV).

Ces dichotomies enragées, qu'inspirait plus la logique de Ramus que celle d'Aristote, permettaient au roi d'être " parfaitement officier " et " parfaitement seigneur ", tout en restant plus officier que seigneur. La seigneurie est " la matière » de la royauté et l'office en est " la forme ", de même que « l'homme est composé de l'âme et du corps ». La doctrine de Loyseau portait en elle une théorie des droits des sujets et une théorie de la fonction publique. La première reposait sur de nouvelles dichotomies : l'éclatement de la seigneurie publique en "souveraine " (celle de l'État) et « suzeraine » (celle des féodaux) et l'éclatement de la seigneurie privée en « directe » (celle des seigneurs des fiefs) et en " utile » (celle des vassaux ou sujets censiers). Ce retour à une vision médiévale d'un imperium et d'un dominium divisés garantissait toutes les libertés et toutes les propriétés. Ainsi se trouvait confortée l'irréductible opposition bodinienne entre "monarchie royale" et "monarchie seigneuriale". La seconde permettait de penser la spécificité de l'office du roi comme union de toutes les formes d'offices et de seigneuries et de le distinguer radicalement de l'office du magistrat. Car, si « l'officier seul se peut qualifier seigneur de son office ", "le roi ou autre collateur de son office [...] est comme le seigneur direct d'icellui » (Offices, I, 2 et Seigneuries, I). La propriété de l'office pouvait donc également être pensée dans la séparation d'une seigneurie directe (celle du roi) et d'une seigneurie utile (celle de l'officier). Le magistrat jouissait d'une propriété qui n'était que « droit à l'office » et non « droit en l'office ». L'indépendance garantie de son droit privé reposait sur la dépendance absolue du droit public qu'il ne tenait que par délégation royale. La modernité de Loyseau s'apprécie mieux quand on songe qu'un aussi fort juriste que Jacques Leschassier avait soumis à Henri IV un Discours de rendre les offices héréditaires et patrimoniaux tenus en fief du Roi.

Le pouvoir royal ne répugnait nullement à entretenir une analogie entre l'office et le fief. L'édit d'octobre 1641 rétablissait les privilèges d' " hérédité " et de " survivance " attribués à certains offices qui, " maintenus héréditaires à perpétuité », tiendront lieu de propres et héritages " en la mouvance de notre seigneurie et hérédité royale ». Même les plus 
absolutistes ne reculaient pas devant une compréhension féodale de la puissance royale. Cardin Le Bret proclamait : « la Couronne de France est un fief masculin", commentaire du titre de alodiis, qui semblerait plutôt impliquer, dans la logique de Le Bret, une assimilation de la couronne à l'alleu, "fief tenu de Dieu et de l'épée ". Il résultait de tous ces raisonnements à base analogique l'émergence de ce qu'on a proposé d'appeler le «dynasticisme propriétaire " ${ }^{7}$. Dans les actes qui concernaient la Bretagne, François $\mathrm{I}^{\text {er }}$ se disait " père légitime, administrateur et usufruitier des biens de notre [...] fils, le dauphin et duc, propriétaire du duché de Bretagne $"{ }^{8}$. Le fils de François $\mathrm{I}^{\text {er }}$ et de Claude de France aurait-il été roi officier en France et duc propriétaire en Bretagne? La substitution du roi aux grands feudataires souligne la parenté étroite qui unit de fait le pouvoir du roi et celui de ses grands vassaux. Le comte Henri de Boulainvilliers, dans sa Lettre sur les Anciens Parlements de France que l'on nomme États Généraux, éditée en 1753, ne disait pas autre chose : « La préséance est due à la noblesse [...] pour la raison décisive et incontestable qu'elle en [il s'agit de l'État] est essentiellement propriétaire, l'ayant conquis par les armes et conservé de même depuis des siècles. " Le droit de conquête, partie intégrante du jus gentium, achevait d'enraciner la royauté dans son passé féodal : Herbert Rowen a pu écrire que le Traité des droits de la Reine très-chrétienne sur divers états de la monarchie d'Espagne (1667), écrit par un groupe d'avocats parisiens pour soutenir les prétentions de Louis XIV sur les Pays-Bas, " renversait d'un trait de plume " toute l'argumentation traditionnelle qui refusait de voir dans la souveraineté un attribut susceptible de propriété. La théorie du domaine royal devait réaliser la fusion, sous le nom de regalia, des droits de souveraineté et des droits seigneuriaux du roi : "le domaine incorporel ne consiste que dans divers droits, dont les uns proviennent de l'exercice de la souveraineté et les autres sont dus au roi en qualité de seigneur des terres [...] dépendantes du domaine de la Couronne ", disait un légiste du $\mathrm{xvII}^{\mathrm{e}}$ siècle $^{9}$. La théorie politique classique a quelque peu oblitéré la nature domaniale et seigneuriale de la puissance royale, elle n'a ni pu ni voulu l'abolir. Il est vrai cependant que l'ombre de la féodalité se projetait de façon de plus en plus oblique sur la souveraineté.

7. Herbert H. Rowen, The King's State. Proprietary Dynasticism in Early Modern France, New Brunswick, Rutgers University Press, 1980, et “ L'État c'est à moi " : Louis XIV and the State ", French Historical Studies, vol. II, 1, 1961, p. 83-98, le livre ne tenant d'ailleurs pas les promesses de l'article.

8. Hélène Michaud, La Grande chancellerie et les écritures royales au xvf siècle (15151589), Paris, Presses universitaires de France, 1967, p. 212.

9. Bosquet, Dictionnaire raisonné des domaines et droits domaniaux, Paris, 1775, t. 1, p. 424. 
Chez C. Loyseau, les théories de l'usurpation et de la prescription s'appliquent aux patrimoines comme aux royaumes : « usurpation suivie d'une longue jouissance fait loi aux souverainetés. " Dans sa Théorie des lois civiles, Simon-Nicolas Linguet reprenait en 1767 cette vieille doctrine en termes provocants : « Les Royaumes appartiennent à leurs Maîtres comme une ferme est à moi [...] Nos titres de jouissance et de propriété sont les mêmes : c'est-à-dire une force, une violence primitive, légitimée ensuite par la prescription. » Les physiocrates ne faisaient-ils pas du roi le premier des propriétaires?

Office et propriété n'étaient nullement antinomiques sous l'ancienne monarchie. La royauté et la magistrature finirent par tourner autour de ces deux pôles sans aucune contradiction interne. Le développement de l'État de finance à l'intérieur de l'État de justice renforçait même les aspects économiques, sinon mercantiles, de l'exercice du pouvoir. Du coup, les notions de seigneurie et de domaine se détachaient de leurs origines féodales et des liens personnels qu'elles impliquaient. On peut discerner dans ce mouvement une modernisation partielle de l'État monarchique. Le commencement de cette mutation politique s'étale sur un long $\mathrm{XvI}^{\mathrm{e}}$ siècle (création des "parties casuelles » en 1523 pour vendre des offices de finance; ordonnance de Moulins en 1566 qui prohibait l'inféodation du domaine et la remplaçait par l'engagement à faculté de rachat ; institution du « droit annuel » (la « paulette ») en 1604 ; développement des « finances extraordinaires " à la faveur de la guerre sous les cardinaux ministres...). Il en résulta un malaise durable qui culmina durant les guerres civiles dans la dénonciation du « machiavélisme ». Louis Charondas Le Caron dans ses Réponses $d u$ droit français, en 1586, fustigeait " les gouverneurs et magistrats qui sont trop épris de l'amour de la République, non du tout pour l'utilité d'icelle, ains plutôt pour la conservation de leur grandeur (qu'on pourrait appeler ambition) ou pour le profit particulier qu'ils y trouvent et en espèrent (qu'aucuns pourraient nommer avarice) ». La vénalité et la fiscalité entraînaient le roi dans le discrédit qui touchait ses officiers. Sans compter, à la même époque, l'aliénation des biens du clergé. Les " antimachiavélistes » accusaient le souverain de défendre « son royaume et son état ", en confondant sa souveraineté avec son domaine et le domaine de la couronne avec sa propriété, deux notions que les juristes, depuis le procureur général La Guesle, sous Henri IV, s'étaient efforcés d'identifier, sans entraîner tout à fait une jurisprudence (celle de la chambre des Comptes) respectueuse des pratiques du " roi dépensier ${ }^{10}$. Bref, le souverain était soupçonné de traiter

10. François Oltvier-Marin, «La réunion de la Basse-Navarre à la couronne de France », Añuario de historia de derecho espanol, vol. IX, 1932, p. 249-289. Yves NevEu, Du 
son office de roi comme les officiers traitaient leurs offices vénaux ${ }^{11} . \mathrm{Ce}$ grand malentendu entre le "bien commun " et les intérêts particuliers des gouvernants parcourut la polémique historique jusqu'à Louis XIV. La théorie de la propriété constituait le point aveugle de la science politique classique.

On peut avancer avec vraisemblance que le roi magistrat était propriétaire de son royaume en un sens archaïque qui se rapproche moins de la propriété quiritaire romaine que de la saisine féodale, cette " possession rendue vénérable par la durée " (Marc Bloch). Le roi de la France moderne restait le seigneur, le dominus. Certains n'avaient pas hésité à tirer du principe ses dernières conséquences : dans le sillage des glossateurs, Barthélemy de Chasseneuz proclamait le $169^{\mathrm{e}}$ des 208 regalia : "Princeps est Dominus omnium rerum sui regni " (Catalogus Gloriae Mundi, 1529). En 1574, Jean Duret, dans L'Harmonie et conférence des magistrats romains avec les officiers français, demeurait fidèle à cette tradition :

«Qui doute que le Prince par un seul rescrit donné de certaine science, ne puisse ôter la possession et seigneurie de l'un pour en revêtir qui bon lui semblera? Ce sont pures rêveries que nos docteurs ont semées quand ils ont cuidé toutes choses leur appartenir [«leur»= les rois] par conservation, non par seigneurie que nous disons domaine."

J. Duret ne veut pas discuter le droit, il se réfère à la pratique, dont l'enseignement principal est que les rois sont généreux :

«Tant s'en faut qu'ils désirent dépouiller le particulier du bien qui lui appartient : il est vrai qu'ils peuvent vendre contre la volonté du maitre l'héritage commun entre lui et le fisc, à la charge de bailler moitié du prix au propriétaire, ou tel autre que sa portion montait."

La directe générale que les rois s'accordaient à eux-mêmes sur les biens des sujets instaurait une co-propriété universelle dont la résultante aurait pu mener au despotisme «à la Turque ».

Quelles étaient donc les "pures rêveries » des docteurs qui s'opposaient à ces thèses "seigneuriales"? Jacques Cujas commentait le Digeste non sans préoccupations d'actualité : il se livrait à une démolition

rôle joué par la Chambre des Comptes de Paris pour assurer le maintien du principe de l'inaliénabilité du domaine, thèse de droit dactylographiée, Paris, 1954. Alain GuERY, "Le roi dépensier. Le don, la contrainte, et l'origine du système financier de la monarchie française d'Ancien Régime ", Annales. E.S.C., 39e année 6, 1984, p. 1241-1269.

11. Salvo MAStellone, Venalità e machiavellismo in Francia (1572-1610). All'origine della mentalità politica borghese, Florence, Olschki, 1972. 
en règle des interprétations des glossateurs, dont Cardin Le Bret dénonçait aussi, sur ce point, la « honteuse et servile flatterie ». Cujas donna en latin - une magistrale réflexion, reprise par François Hotman, sur les "biens du prince » («bona principis »), qui sont, soit droits fiscaux ("fiscalia »), soit droits patrimoniaux (" patrimonialia »). C'est ainsi qu'il faut entendre l'adage stoïcien : «Omnia esse principis. » Car les droits du prince ne répondent pas à un principe unique :

«Ce que les particuliers possèdent est sous l'imperium du prince. Les biens propres (" bona propria") que possède le prince sont dans le patrimoine et la seigneurie (dominium) du prince. Les droits du fisc (fiscalia) sont quasi dans le patrimoine du prince et les propriétés privées (proprie) ne sont pas dans son patrimoine. "

Cette analyse aboutit au double adage : « ut omnia Rex imperio possideat, singuli dominio » et "omnia esse Principis imperio : singulorum enim dominio " (Paratitla in libros IX Codicis Justiniani, I, XXXIII). Tel était, pour Cujas, l'enseignement du Codex Justiniani, du Digeste et de Sénèque. Une telle théorie impliquait une séparation au moins relative entre un règne du privé, celui du dominium, et un règne du public, celui de l'imperium. Cujas retrouvait le postulat fondamental de la philosophie politique de Bodin ou de Loyseau. Mais il faut dire qu'il en tirait des conséquences franchement hostiles aux théories absolutistes qu'il condamnait nettement par ailleurs : " aujourd'hui les princes ne sont pas absolus, ce qui est très certain, puisqu'ils jurent fidélité aux lois de la patrie (" leges patrias "), qu'ils ne juraient pas autrefois ", n'hésitait-il pas à écrire, usant d'un argument qui ne valait pas très cher.

Il y eut un temps où la monarchie professait des thèses similaires à celles de Cujas : " Je ne voudrais, disait le chancelier Michel de L'Hospital, le 13 décembre 1560, que les rois estimassent que les biens de leursdits sujets leur appartiennent imperio, non dominio et proprietate. " La plupart des juristes interprétaient dans le même sens le De Officiis de Sénèque, par exemple, Loyseau qui évoquait « la seigneurie universelle que le roi a sur tous les biens de ses sujets » et citait Sénèque : «Principis omnia sunt imperio, non dominio. " Et tous en tiraient la même conclusion pratique que L'Hospital : inviter le souverain à se contenter des revenus de son domaine, à ne pas charger le peuple de tailles, pour que, concluait le chancelier, « les sujets l'aimassent et reconnussent pour roi et seigneur, l'aidassent de leurs personnes et biens ». Le recours à une terminologie romaine habillait une vision traditionnelle imprégnée d'esprit féodal : "le roi doit vivre du sien "; le peuple fournit des "dons gratuits " pour « aider » son roi seigneur. Dans cette perspective, l'impôt, justifié par le bien public, ne devait-il pas être consenti? La monarchie 
avait répondu par la négative à cette question cruciale, mais les jurisconsultes continuaient à s'interroger : Loyseau corrigeant, en 1608 , les épreuves de son Traité des seigneuries, se prononçait dans une prudente formule, contre la nécessité du consentement de l'impôt. Mais c'était aussi par prescription que le roi avait acquis ce droit de « faire levées de deniers sans le consentement des états". Bodin affirmait que "les charges et impositions sur les sujets sont très justes : car il n'y a rien plus juste que ce qui est nécessaire ", mais il condamnait la " détestable invention " de Denys le Tyran qui cherchait des occasions de faire la guerre pour établir de nouveaux impôts qu'il continuerait après la paix (accusations qui furent portées contre Richelieu et Mazarin). Bodin penchait pour le consentement de l'impôt (République, VI, 2), alors qu'il rejetait celui de la loi. Cardin Le Bret n'éprouvait plus d'hésitation quant à la légitimité de l'impôt royal, mais il l'assortissait de restrictions morales et d'une référence formelle au principe «le roi doit vivre du sien » :

« Depuis que la guerre, comme un feu dévorant, a consommé la plupart du fond de leur domaine, [les rois] ont été contraints d'user absolument de leur autorité et de lever sur leurs peuples des tailles et des subsides, même sans leur consentement, qui est un des droits les plus remarquables de la souveraineté des rois...»

Cette théorie requérait une limitation du droit de propriété : « en certaines circonstances, le Prince [peut] contraindre les particuliers d'abandonner leurs biens au public ", d'autant, notait Le Bret, que les héritages privés ont été " autrefois communs et publics " (De la souveraineté $d u$ roi, IV, 10-11). La notion de nécessité mise en relation avec le bien public, l'avait donc radicalement emporté sur l'idée de consentement

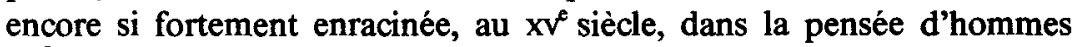
d'État comme Jean Juvénal des Ursins.

Deux justifications voisines venaient légitimer ces droits du roi. La première, d'esprit féodal, était la théorie de la "directe royale universelle " qui, entre autres, ruinait le précepte méridional et romain de la « liberté des francs alleux " : le roi est " seigneur universel de toutes les terres qui sont dans son royaume", sauf privilèges contraires. Imitant le « code Michau " de 1629, l'édit d'août 1692 généralisa ce principe. Louis XIV proclamait : « nous n'avons point de droit ni mieux établi, ni plus inséparablement attaché à notre Couronne que celui de la mouvance et directe universelle... $"{ }^{12}$. Louis XIV ne se contentait pas que tous les Français

12. Voir Émile Chenon, Etude sur l'histoire des alleux en France, Paris, 1888 ; Ed. ANDT, "Théorie de la directe universelle présentée d'après l'édit de 1692 », Revue historique de droit français et étranger, 1922, p. 604-636. 
fussent ses sujets, il voulait encore qu'ils fussent ses vassaux. La seconde, à connotations sacerdotales, était la notion de "domaine éminent" à bien distinguer du "domaine ordinaire", quoique l'un et l'autre aient " leur fondement dans la puissance absolue pour le bon gouvernement". "L'étendue et l'usage " du domaine éminent «sont déterminés par les besoins de l'État". Ainsi "le souverain de chaque pays a droit sur les terres et autres biens des sujets [...] en trois matières principalement" : « régler par les lois l'usage que chacun en doit faire »; « exiger les impôts pour les charges de l'État »; " exiger les droits du domaine éminent dans les occasions qui se présentent " (François de Paule Lagarde, Traité historique de la souveraineté $d u$ roi..., 1754).

L'universalité de la propriété royale était ancrée, comme la souveraineté, dans la notion traditionnelle du bien commun. Les proclamations de Louis XIV, le plus sensible des rois à la tentation « tyrannique ", ne se référaient finalement qu'à une vision autoritaire de l'intérêt général :

“Tout ce qui se trouve dans l'étendue de nos États, de quelque nature qu'il soit, nous appartient au même titre : les deniers qui sont dans nos cassettes, ceux qui demeurent entre les mains des trésoriers et ceux que nous laissons dans le commerce de nos peuples, doivent être par nous également ménagés. "

Louis XIV s'engageait-il de la sorte à se montrer bon ménager de la monnaie que les rois seuls avaient le droit de battre? L'identité de ce «titre" de possession s'étendait aux autres natures de bien : «les rois sont seigneurs absolus et ont naturellement la disposition pleine et libre de tous les biens, tant séculiers que des ecclésiastiques, pour en user comme de sages économes, c'est-à-dire selon les besoins de leur État » (Supplément aux Mémoires et Mémoires pour 1666). Dans cette vision extensible des droits du roi, l'impôt n'avait plus à être justifié et la référence au consentement sentait son crime de lèse-majesté. En même temps, le roi partageant les intérêts de ses sujets propriétaires s'efforcera nécessairement, selon les physiocrates, de défendre le droit naturel. Il est indéniable que tous les rois, y compris Louis XIV, respectèrent la propriété des sujets. Les France-Turquie et autres allégations contraires (par exemple dans les Maupeouana en 1771) relèvent de l'artifice polémique.

En connaissance de cause, la monarchie a favorisé le développement de la propriété : elle créa même de nouveaux types de biens immeubles gagés pour ainsi dire sur l'État. François $\mathrm{I}^{\mathrm{er}}$ joua en la matière un rôle de pionnier, puisqu'il créa, en 1522-1523, à la fois, les rentes constituées sur l'Hôtel de ville et les " finances " d'offices vénaux. Les rentes finirent par être garanties sur toutes sortes d'impôts, non seulement les aides et les gabelles, mais aussi les tailles (rentes sur les recettes générales), et le 
clergé consentit finalement à aliéner une partie de son temporel pour s'associer au financement des guerres de Religion (contrat de Poissy, 1561). Les rentes, sinon les rentiers, connurent une fortune qui ne se démentit pas durant tout l'Ancien Régime. La vénalité des offices conférait aux officiers un droit sur le trésor public : sauf forfaiture, on ne pouvait supprimer l'office sans rembourser aux héritiers ce qui avait été versé aux coffres du roi. $\mathrm{Au}$ xvil ${ }^{\mathrm{e}}$ siècle, le système donna lieu à une spéculation à grande échelle, d'autant que les offices créés étaient souvent mis en " partis », c'est-à-dire cédés en gros à des financiers qui se chargeaient de trouver des acheteurs. Le processus de construction de l'État moderne multipliait ainsi les créanciers de l'État. Rentiers, officiers, financiers n'avaient pas bénéficié de la "grâce " du monarque, ils lui avaient (ou bien étaient censés lui avoir) rendu des services pécuniaires qui donnaient naissance à une situation contractuelle. «On ne marchande pas avec le roi », disait Louis XIII à un premier président du parlement qui négociait le rappel de conseillers exilés. Mais quand le roi avait lui-même marchandé et accepté l'argent de ses sujets, il était tenu à ses conventions " religieusement ", à la fois " pour l'équité naturelle » et « pour la foi du prince qu'il doit tenir, ores qu'il y eût dommage, parce qu'il est garant formel à tous ses sujets de la foi qu'ils ont entre eux ".

«C'est pourquoi, continue Bodin idéalisant quelque peu une pratique plus ambiguë, le Prince souverain doit être toujours moins supporté en justice que ses sujets, quand il y va de sa promesse : car il ne peut ôter l'office donné à son sujet sans juste cause : et le seigneur particulier le peut faire [...] et si ne peut ôter le fief à son vassal sans cause, les autres seigneurs le peuvent par les maximes des fiefs » (République, I, 8).

Le dernier exemple choisi par Bodin illustre bien les racines féodales de la fidélité du prince. Mais la croissance parallèle de l'État et de l'économie monétaire avait enserré la monarchie dans tout un réseau de liens contractuels de nature pécuniaire et donc plus moderne. Les rois purent bien, de banqueroutes partielles en mutations monétaires, de baisses du taux d'intérêt en créations d'offices à bas prix, rogner les créances des sujets, ils n'en nièrent jamais la source et le principe ${ }^{13}$.

Tels sont les fondements les plus neufs et les plus solides de l'« État de droit » monarchique. La " modernité " politique du xvie siècle réside là. Elle s'était elle-même bâtie sur la tradition médiévale du roi comme sei-

13. Sur les usages, voir Françoise Bayard, Le Monde des financiers au xvif siècle, Paris, Flammarion, 1988; sur les effets de la monétarisation, voir Marie-Thérèse BoYer-XaMBEU, Ghislain DelePlace, Lucien Gillard, Monnaie privée et pouvoir des princes. L'économie des relations monétaires à la Renaissance, Paris, Presses de la Fondation nationale des sciences politiques/Ed. du C.N.R.S., 1986. 
gneur justicier. Les jurisconsultes insistent fort sur la facilité dont jouissaient les Français pour réclamer leurs droits contre le roi. Les cours souveraines n'hésitèrent jamais à condamner les procureurs généraux au nom du roi. Mais G. Chevrier ne s'y trompait pas, qui rappelait qu' « une conception absolutiste du pouvoir ne permettait guère à un particulier de revendiquer des droits vis-à-vis de l'État, hormis le cas où il avait passé contrat avec celui-ci ou réclamait la protection d'un droit acquis $"{ }^{14}$. En effet, toute loi et tout privilège étant enveloppés dans la volonté législatrice du monarque, on ne revendiquait pas de droits, on sollicitait - par placets - des "grâces » que distribuait à foison le « roi dépensier ». Mais l'État, lui, était un enchevêtrement de droits contractuels acquis par ses serviteurs empressés à se servir au service du souverain. Le conseil d'État jugeait principalement des conditions d'application de ces contrats. L'État s'y trouvait juge et partie : les constructeurs d'État moderne puisaient dans ces arbitrages indulgents la force de continuer l'œuvre.

Il n'est pas vrai que le roi de France ne considéra « que son droit de propriété sur les Etats " qu'il gouvernait. Mais si, pour continuer à paraphraser la formule de Voyer d'Argenson, il se regardait comme le premier magistrat, il n'oubliait pas néanmoins que le royaume lui appartenait en un sens. Cette royale propriété qui ne s'assimilait pas à une propriété privée, avait essaimé, sous forme d'offices, des milliers de petites quasipropriétés à son image. Jusqu'à œ que Colbert et Louis XIV manifestent la relative désaffection de la monarchie à l'égard de l'office et des officiers, une solidarité reposant sur l'esprit propriétaire avait réuni tous les détenteurs d'offices, depuis le souverain jusqu'au moindre juge ou financier investi d'une jurisdictio ou d'une potestas. Le roi, en considérant sa seigneurie éminente sur le royaume, regardait avec faveur les autres seigneurs. Le caractère archaïque et féodal de ses propres droits le poussait à la compréhension à l'égard des autres propriétaires féodaux, pourvu naturellement qu'ils respectassent sa souveraineté et supériorité. Ce même terme qualifiait souvent encore le pouvoir du roi et celui des seigneurs.

Mais l'évolution sociale et le succès historique des nouvelles formes de propriété conduisaient déjà Robert-Joseph Potier à reléguer le droit des seigneurs en un « domaine de supériorité » qui n'impliquait plus guère que des droits recognitifs laissant intactes les prérogatives propriétaires des censiers (Traité du droit de domaine de propriété, 1772). À n'en pas

14. Georges Chevrier, « Remarques sur l'introduction et les vicissitudes de la distinction du jus privatum et du jus publicum dans les cuvres des anciens juristes français ", Archives de philosophie du droit, nouvelle série, vol. 1, 1952, p. 9-10. 
douter, en ce siècle, le dominium du roi ne cessa de s'étioler aussi. Le retour en force de la conception romaine quiritaire de la propriété - y compris l'abusus - ne pouvait gagner le royaume dont la fin restait le bien commun. C'était toute la signification de la théorie statutaire mise au point depuis Terrevermeille. Mais la modernisation des relations et des idées sociales n'avança que lentement, et il n'est pas sûr que la monarchie agit toujours dans le sens du changement. Toutes les formes de propriétés étaient solidaires. On ne pouvait pas sans révolution préserver les unes pour supprimer les autres. On ne pouvait pas sans despotisme s'attaquer à la propriété seigneuriale. Au demeurant, la royauté n'eut jamais beaucoup de velléités en ce sens et la simple idée d'abolir les justices des seigneurs se heurta toujours à cette exigence fondatrice. L'appropriation du pouvoir royal garantissait l'unité de la propriété. «Que de personnages, s'exclamait Marc Bloch décrivant la multiplicité des droits seigneuriaux, qui, avec autant de raison l'un que l'autre, peuvent dire "mon champ"! " 15 À l'âge moderne, le roi vit renforcer ses droits omniprésents. La France n'en devint pas pour autant une «monarchie seigneuriale ". Il est très frappant que Loyseau ait proposé imperium comme un des équivalents latins de ce qu'il nommait la « seigneurie publique " qui, à ses yeux, n'impliquait même pas très clairement le droit de lever les impôts. Rien ne montre mieux que la féodalité et la confusion qu'elle entretenait entre le dominium et l'imperium n'étaient pas plus mortes dans les têtes que dans les faits au temps de la monarchie absolue.

Ainsi la loi fondamentale (concept qui prend naissance par la plume de Théodore de Bèze), sous ses deux aspects, dévolution statutaire et inaliénabilité du domaine, paraît une solution élégante pour régler les rapports transitifs du dominium et de l'imperium et les rapports immanents entre la puissance du roi magistrat et les pouvoirs du roi seigneur. Mais le « roi fiscal » et le « roi féodal », pour reprendre une terminologie qui est celle d'Ernst Kantorowicz, ne sont pas des figures étrangères l'une à l'autre.

En premier lieu, les deux figures du roi se conforment à un même schéma formel du pouvoir, celui qu'exprime la polysémie des termes dominus, dominium et domus, schéma qui peut être pensé comme une « structure » à la Lévi-Strauss ou comme un «énoncé » à la Foucault.

15. Marc Bloch, La Société féodale, 1939, Paris, Albin Michel, «L'Évolution de l'humanité $", 1968$, p. 173-175. Cette parole pourrait être étendue aux seigneuries et aux dignités : qui du seigneur ou du vassal doit-il être dit " propriétaire "? On peut encore lire les analyses d'un auteur très prisé par les historiens du droit, Paul LuCAs-ChaMPIonNière, De la propriété des eaux courantes..., ouvrage contenant l'exposé complet des institutions seigneuriales, Paris, 1846 , p. 146-150. 
Dieu, le roi, le seigneur féodal, l'officier public, l'évêque ou l'abbé et encore le paterfamilias se trouvent dans une position symétrique par rapport à l'univers (ou à l'humanité), au royaume (ou aux sujets), à la seigneurie (ou aux vassaux), à l'office (ou aux justiciables), à l'église (ou aux fidèles) ou à l'abbaye (ou aux moines), sans oublier la maison (ou famille)... L'objet de l'autorité impartie est toujours une abstraction qui subsume une collectivité humaine (une universitas?); et les modalités en sont fixées par un système de normes transcendantes (théologie, droits divin, féodal, public, canon, naturel...) et transmises à travers un rituel qui implique mystère et serment (sacre, investiture, provision, sacrement du mariage...). L'objet de cette relation de puissance ne peut être qu'un accomplissement essentiel qui comporte dans sa logique intrinsèque un ministerium (divinité, royauté, seigneurie, magistrature, bénéfice séculier ou régulier, autorité paternelle...) et des rapports d'amour, sanction de la foi ou fidélité exaltée par le rituel. Découvreur de ce schéma formel, dont il explore inconsciemment l'universalité, Charles Loyseau (Offices, II, 2, \$21-28) fait du souverain un « officier et feudataire tout ensemble à l'égard de Dieu et à l'égard du Peuple " et remarque que «le Roy de France et les autres Roys qui ont accoustumé d'estre sacrés, ont tout ensemble les trois espèces de Dignitez, l'ordre, l'office, et la seigneurie ». Ce qui fonde la souveraineté du " roi fiscal " (fiscus, Reipublicae anima, dit Balde), n'est-ce donc pas la curatelle exercée sur l'utilité publique, grande justification de l'inaliénabilité de la souveraineté et du domaine? Ce qui fonde la souveraineté du « roi féodal ", n'est-ce pas qu'elle est la "vive image de la divine" (Jean Savaron, De la souveraineté du Roi, 1620)?

En second lieu, le roi, « seigneur central " (Norbert Élias), coincé entre les princes ecclésiastiques et les grands feudataires au Moyen Âge, entre le haut clergé, la haute noblesse et la haute robe d'État dans les Temps modernes, construit ses pouvoirs concrets sur le contrôle et l'action d'appareils : hiérarchie descendante des fidélités féodales que le souverain surplombe, d'abord; et pyramide de l'État moderne, façonnée sur un modèle féodal élargi, ensuite. Une comparaison formelle entre les deux appareils fait ressortir parallélismes et dissemblances qui mettent en lumière l'affinité génétique unissant le "roi féodal " et le "roi fiscal ". Historiquement, le fonctionnement des appareils socio-politiques produit les limitations réelles, intrinsèques mais efficaces, qui bornent le pouvoir monarchique, limitations qu'il importerait de comparer ou de rapporter aux théories politiques qui enferment la souveraineté dans les frontières logiques de son essence.

À ce stade, il faudrait, pour comprendre la culture politique de l'ancienne monarchie, faire intervenir l'étude des cérémonials qui, dans 
leur quasi-liturgie, réconcilient les inconciliables de la pensée et de l'action. Mais, même sans cette clef, les rapprochements esquissés n'interdisent-ils pas de penser le roi seigneur en termes d'archaïsme et de despotisme et le roi officier en termes de modernité et de liberté ?

Robert DESCIMON, Centre de recherches historiques, École des hautes études en sciences sociales. 